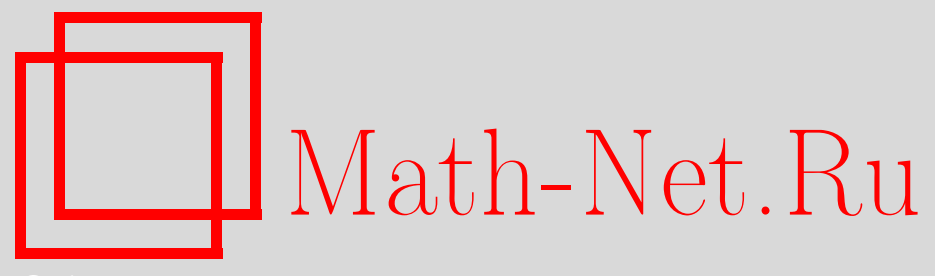

Ю. М. Стреляев, Метод нелинейных граничных интегральных уравнений для решения квазистатической контактной задачи о взаимодействии упругих тел при наличии кулонова трения, Becmн. Сам. гос. техн. ун-та. Сер. Физ.мат. науки, 2016, номер 2, 306-327

DOI: https://doi.org/10.14498/vsgtu1471

Использование Общероссийского математического портала MathNet.Ru подразумевает, что вы прочитали и согласны с пользовательским соглашением

http://www.mathnet.ru/rus/agreement

Параметры загрузки:

IP: 52.23 .180 .231

26 апреля 2023 г., 16:41:20

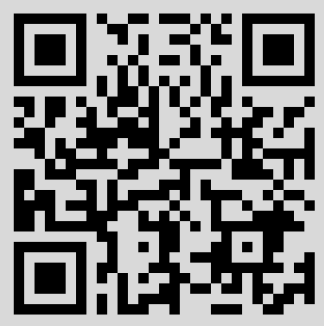


УДК 539.3

\title{
МЕТОД НЕЛИНЕЙНЫХ ГРАНИЧНЫХ ИНТЕГРАЛЬНЫХ УРАВНЕНИЙ ДЛЯ РЕШЕНИЯ КВАЗИСТАТИЧЕСКОЙ КОНТАКТНОЙ ЗАДАЧИ О ВЗАИМОДЕЙСТВИИ УПРУГИХ ТЕЛ ПРИ НАЛИЧИИ КУЛОНОВА ТРЕНИЯ
}

\author{
Ю. М. Стреляев
}

Запорожский национальный университет,

Украина, 69600, Запорожье, ул. Жуковского, 66.

\begin{abstract}
Аннотация
Рассмотрена пространственная квазистатическая контактная задача о взаимодействии двух линейно-упругих тел с учетом кулонова трения между ними. В граничных условиях задачи на каждом шаге дискретного процесса нагружения были приняты упрощения, основанные на модификации закона трения Кулона. Эта модификация состояла во введении в соотношения, выражающие закон трения Кулона, запаздывания контактных давлений, ограничивающих касательные контактные напряжения. В рассмотренной постановке задача бала сведена к последовательному решению серии однотипных систем нелинейных интегральных уравнений, описывающих взаимодействие тел на каждом из шагов нагружения. Для получения контактных напряжений на каждом шаге нагружения использован метод приближённого решения системы интегральных уравнений этого шага, который заключается в регуляризации этой системы уравнений, дискретизации регуляризированной системы и применении сходящегося итерационного процесса для получения решения дискретизированной системы. Предложенным методом получено численное решение контактной задачи о вдавливании упругого шара в упругое полупространство при возрастании и последующем убывании нормальной сжимающей силы.
\end{abstract}

Ключевые слова: упругое тело, контактная задача, трение Кулона, квазистатическая задача, интегральное уравнение, единственность решения, регуляризирующее уравнение, итерационный процесс.

Введение. Для решения контактных задач теории упругости с учетом трения часто используются численные методы, основанные на вариационной постановке задачи $[1,2]$ либо на её сведении к различным нелинейным операторным уравнениям [3-7]. Основная трудность реализации вариационных методов заключается в необходимости рассматривать сложные задачи нелинейного программирования. Иногда эту трудность удается устранить путем

(C) 2016 Самарский государственный технический университет.

\section{Образец для цитирования}

С треля в Ю. М. Метод нелинейных граничных интегральных уравнений для решения квазистатической контактной задачи о взаимодействии упругих тел при наличии кулонова трения // Вестн. Сам. гос. техн. ун-та. Сер. Физ.-мат. науки, 2016. Т. 20, № 2. С. 306327. doi: $10.14498 /$ vsgtu1471.

\section{Сведения об авторе}

Юрий Михайлович Стреляев (strelkiny@gmail.com), старший преподаватель, каф. математического анализа. 
использования нелинейных операторных уравнений для моделирования контактного взаимодействия тел. Однако в большинстве случаев при использовании таких уравнений авторы либо не учитывают трение [3-5], либо его учет осуществляется при упрощенных граничных условиях, соответствующих полному проскальзыванию тел [6,7]. Хотя метод нелинейных граничных интегральных уравнений, предложенный в работе [8], и позволяет рассматривать закон трения Кулона в сложном классическом виде, но при использовании этого метода трудно выразить условия единственности решения контактной задачи и доказать сходимость итерационных процессов, применяемых для получения этого решения [9]. В настоящей статье предложена модификация нелинейных граничных интегральных уравнений [8], позволяющая избавиться от указанных трудностей. Данная модификация основана на упрощении граничных условий квазистатической контактной задачи на каждом шаге процесса нагружения тел. Это упрощение позволяет свести контактную задачу к последовательному решению серии однотипных нелинейных операторных уравнений второго рода с квазисжимающим оператором в правой части. Такая особенность этих уравнений позволяет разработать эффективный метод их приближённого решения. Целью данной статьи является разработка такого метода и получение строгого математического обоснования основных его этапов.

1. Постановка контактной задачи. Предположим, что каждое из двух тел на некоторой части своей поверхности сцеплено с абсолютно твёрдым телом, которое будем называть жесткой опорой. Первоначально касаясь в одной точке, тела входят в контакт за счет того, что опора верхнего тела совершает заданное поступательное перемещение $\gamma(t)$, зависящее от времени $t$, в то время как опора нижнего тела остается неподвижной. Требуется найти поверхность соприкасания тел после установившего равновесия и распределение контактной нагрузки на этой поверхности. Относительно взаимодействующих тел принимаются следующие допущения:

1) оба тела являются линейно-упругими и изотропными;

2) упругие перемещения точек малы по сравнению с размерами поверхности контакта;

3) в каждой точке поверхности каждого из тел в зоне возможного контакта существует единственная касательная плоскость к этой поверхности;

4) операторы влияния поверхностных нагрузок на поверхностные упругие перемещения для каждого из тел являются известными и допускают интегральное представление.

Будем считать, что вектор-функция $\gamma(t)$ медленно изменяется с возрастанием $t$ от 0 до фиксированного значения $T>0$, и пренебрегать инерционными и волновыми эффектами. Предположим, что процесс взаимодействия тел сопровождается поверхностным трением, подчиняющемся закону Кулона [10]. Будем ещё полагать, что в любой момент времени $t>0$ поверхность соприкасания тел является плоской и содержится в известной ограниченной области $\Omega$, принадлежащей общей для тел касательной плоскости П, проходящей через точку их начального касания. Граница областей сцепления и проскальзывания заранее не известна и подлежит определению в ходе решения. Введем прямоугольную систему координат $O z x y$ с началом в точке первоначального касания тел и осью $z$, направленной внутрь верхнего тела 
перпендикулярно плоскости П.

Будем рассматривать процесс нагружения тел лишь в фиксированные моменты времени $t_{0}, t_{1}, t_{2}, \ldots, t_{l}$ такие, что $0=t_{0}<t_{1}<t_{2}<\cdots<t_{l}=T$. Будем также предполагать, что при каждом значении $t=t_{i}$ для системы контактирующих тел устанавливается равновесие. Таким образом, процесс нагружения тел происходит пошагово и состоит из конечного числа $l$ последовательных состояний равновесия. Пусть индекс $i$ определяет номер шага нагружения и вектор-функция $\gamma(t)$ принимает на каждом шаге нагружения значение $\gamma_{i}=\gamma\left(t_{i}\right)=\left(\Delta_{1 i}, \Delta_{2 i}, \Delta_{3 i}\right)$ (рис. 1$)$.

Граничные условия контактного взаимодействия тел на $i$-том шаге нагружения будем выражать с помощью двух вектор-функций

$$
p_{i}(s)=\left(p_{1 i}(s), p_{2 i}(s), p_{3 i}(s)\right), \quad v_{i}(s)=\left(v_{1 i}(s), v_{2 i}(s), v_{3 i}(s)\right),
$$

где $s=(x, y)$ - произвольная точка области $\Omega$, определяющая пару противолежащих точек (рис. 1) на поверхностях взаимодействующих тел (предполагается, что произвольная точка поверхности верхнего тела может вступить в контакт только с противолежащей точкой поверхности нижнего тела). Вектор-функция $p_{i}(s)$ определяет распределение удельной контактной нагрузки, передаваемой от нижнего тела к верхнему телу через точки области $\Omega$ на $i$-том шаге нагружения. Вектор-функция $v_{i}(s)$ задает перемещения точек поверхности верхнего тела относительно противолежащих точек поверхности нижнего тела на $i$-том шаге нагружения. Зависимость компонент этих вектор-функций от времени выражается лишь в дискретном изменении их значений при переходе к следующему шагу нагружения. Отметим, что значения $1,2,3$ индексов соответствуют направлениям координатных осей $O z, O x, O y$. С учетом принятых предположений граничные условия контактного взаимодействия тел можно выразить следующей системой неравенств и уравнений:

$$
\begin{aligned}
& p_{1 i}(s) \geqslant 0 ; \quad v_{1 i}(s) \geqslant 0 ; \quad p_{1 i}(s) v_{1 i}(s)=0 ; \\
& \sqrt{p_{2 i}^{2}(s)+p_{3 i}^{2}(s) \leqslant \mu p_{1 i}(s) ;} \\
& \sqrt{\left[\dot{v}_{2 i}(s)\right]^{2}+\left[\dot{v}_{3 i}(s)\right]^{2}} p_{2 i}(s)+\mu p_{1 i}(s) \dot{v}_{2 i}(s)=0 ; \\
& \sqrt{\left[\dot{v}_{2 i}(s)\right]^{2}+\left[\dot{v}_{3 i}(s)\right]^{2}} p_{3 i}(s)+\mu p_{1 i}(s) \dot{v}_{3 i}(s)=0
\end{aligned}
$$

где $s \in \Omega, i=\overline{1, l}$; положительная константа $\mu$ - коэффициент трения, а $\dot{v}_{2 i}(s)$, $\dot{v}_{3 i}(s)$ - компоненты вектора скорости относительного проскальзывания тел в точке $s \in \Omega$ на $i$-том шаге нагружения.

Первое из соотношений (1) выражает знакопостоянство контактного давления, второе означает отсутствие взаимного проникания тел, третье означает отсутствие контактного давления за пределами площадки контакта. Последние три соотношения системы (1) выражают закон трения Кулона.

Будем считать, что компоненты $p_{k i}, v_{k i}$ вектор-функций $p_{i}(s), v_{i}(s)$ принадлежат гильбертову пространству $L_{2}(\Omega)$, а сами вектор-функции $p_{i}(s)$, $v_{i}(s)$ являются элементами гильбертова пространства $L_{2}^{3}(\Omega)$ [9]. Связь между вектор-функциями $p_{i}(s)$ и $v_{i}(s)$ выражается соотношениями

$$
v_{k i}(s)=\sum_{j=1}^{3} A_{k j}\left(p_{j i}\right)_{s}-f_{k i}(s) \equiv F_{k}\left(p_{i}, \Delta_{k i}\right)_{s} \forall k=\overline{1,3},
$$




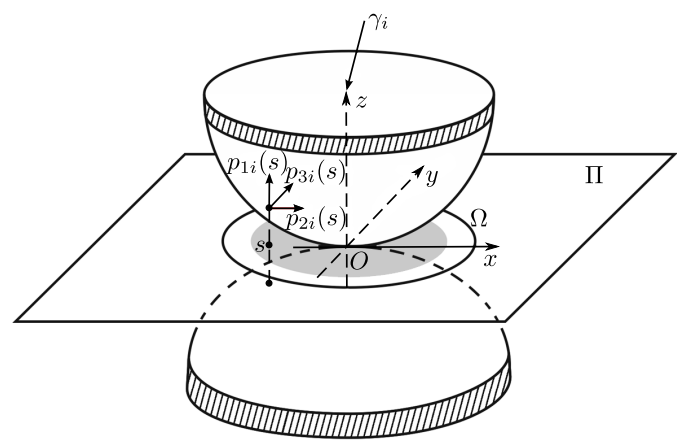

Рис. 1. Схема контактного взаимодействия упругих тел

[Figure 1. The scheme of contact interaction of elastic bodies]

где $A_{k j}: L_{2}(\Omega) \rightarrow L_{2}(\Omega)$ - операторы влияния поверхностных нагрузок на поверхностные упругие перемещения; $f_{i}(s)=\left(f_{1 i}(s), f_{2 i}(s), f_{3 i}(s)\right) \in L_{2}^{3}(\Omega)-$ известная вектор-функция, для которой $f_{1 i}(s)=-\delta_{0}(s)-\Delta_{1 i}, f_{2 i}(s)=-\Delta_{2 i}$, $f_{3 i}(s)=-\Delta_{3 i}$, а функция $\delta_{0}(s) \in L_{2}(\Omega)$ задает первоначальный зазор между телами, определяемый уравнениями их поверхностей. Выразим компоненты $\dot{v}_{2 i}(s), \dot{v}_{3 i}(s)$ вектора скорости относительного проскальзывания тел в точке $s \in \Omega$ на $i$-том шаге нагружения с помощью конечных разностей:

$$
\dot{v}_{k i}(s)=\frac{v_{k i}(s)-v_{k i-1}(s)}{h_{i}}=\frac{1}{h_{i}} F_{k}\left(p_{i}, \tilde{\Delta}_{k i}\right)_{s}, \quad i=\overline{1, l}, k=2,3,
$$

где $h_{i}=t_{i}-t_{i-1}, \tilde{\Delta}_{k i}(s)=\Delta_{k i}-F_{k}\left(p_{i-1}, \Delta_{k i-1}\right)_{s}$, а $F_{k}\left(p_{i}, \Delta_{k i}\right)_{s}$ определяется соотношениями (2). Тогда систему (1) можно записать в виде

$$
\begin{aligned}
& p_{1 i}(s) \geqslant 0 ; \quad F_{1}\left(p_{i}, \Delta_{1 i}\right)_{s} \geqslant 0 ; \quad p_{1 i}(s) F_{1}\left(p_{i}, \Delta_{1 i}\right)_{s}=0 ; \\
& \sqrt{p_{2 i}^{2}(s)+p_{3 i}^{2}(s)} \leqslant \mu p_{1 i}(s) ; \\
& \sqrt{F_{2}^{2}\left(p_{i}, \tilde{\Delta}_{2 i}\right)_{s}+F_{3}^{2}\left(p_{i}, \tilde{\Delta}_{3 i}\right)_{s}} p_{2 i}(s)+\mu p_{1 i}(s) F_{2}\left(p_{i}, \tilde{\Delta}_{2 i}\right)_{s}=0 ; \\
& \sqrt{F_{2}^{2}\left(p_{i}, \tilde{\Delta}_{2 i}\right)_{s}+F_{3}^{2}\left(p_{i}, \tilde{\Delta}_{3 i}\right)_{s}} p_{3 i}(s)+\mu p_{1 i}(s) F_{3}\left(p_{i}, \tilde{\Delta}_{3 i}\right)_{s}=0 ;
\end{aligned}
$$

где $s \in \Omega, i=\overline{1, l}$.

Таким образом, рассматриваемая контактная задача на $i$-том шаге нагружения состоит в определении неизвестных функций $p_{k i}(s) \in L_{2}(\Omega), k=\overline{1,3}$, которые почти всюду на $\Omega$ удовлетворяют соотношениям (4). Отметим, что из соотношений (3) следует, что для нахождения решения системы (4) на $i$-том шаге нагружения должно быть известно решение этой системы на $(i-1)$-м шаге. В качестве начальных условий на нулевом шаге (в момент начального касания тел) примем тождественное равенство нулю всех компонент векторфункций $\gamma_{0}$ и $p_{0}(s)$.

Для упрощения постановки контактной задачи рассмотрим следующую модификацию соотношений, выражающих закон трения Кулона в системе (4). 
Вначале запишем эту систему в эквивалентном виде:

$$
\begin{aligned}
& p_{1 i}(s) \geqslant 0 ; \quad F_{1}\left(p_{i}, \Delta_{1 i}\right)_{s} \geqslant 0 ; \quad p_{1 i}(s) F_{1}\left(p_{i}, \Delta_{1 i}\right)_{s}=0 ; \\
& \sqrt{p_{2 i}^{2}(s)+p_{3 i}^{2}(s)} \leqslant \mu\left(p_{1 i-1}(s)+\varepsilon_{i}(s)\right) ; \\
& \sqrt{F_{2}^{2}\left(p_{i}, \tilde{\Delta}_{2 i}\right)_{s}+F_{3}^{2}\left(p_{i}, \tilde{\Delta}_{3 i}\right)_{s}} p_{2 i}(s)+\mu\left(p_{1 i-1}(s)+\varepsilon_{i}(s)\right) F_{2}\left(p_{i}, \tilde{\Delta}_{2 i}\right)_{s}=0 ; \\
& \sqrt{F_{2}^{2}\left(p_{i}, \tilde{\Delta}_{2 i}\right)_{s}+F_{3}^{2}\left(p_{i}, \tilde{\Delta}_{3 i}\right)_{s}} p_{3 i}(s)+\mu\left(p_{1 i-1}(s)+\varepsilon_{i}(s)\right) F_{3}\left(p_{i}, \tilde{\Delta}_{3 i}\right)_{s}=0,
\end{aligned}
$$

где $s \in \Omega, i=\overline{1, l} ; \varepsilon_{i}(s)=p_{1 i}(s)-p_{1 i-1}(s)$. Затем будем предполагать, что при достаточно большом числе шагов нагружения $l$ разности $p_{1 i}(s)-p_{1 i-1}(s)$ малы по сравнению с величинами контактных давлений $p_{1 i}(s)$ на каждом $i$-том шаге нагружения. Наконец, учитывая последнее предположение, отбросим в системе (5) все приращения $\varepsilon_{i}(s)$. Тогда система (5) примет следующий модифицированный вид:

$$
\begin{aligned}
& p_{1 i}(s) \geqslant 0 ; \quad F_{1}\left(p_{i}, \Delta_{1 i}\right)_{s} \geqslant 0 ; \quad p_{1 i}(s) F_{1}\left(p_{i}, \Delta_{1 i}\right)_{s}=0 ; \\
& \sqrt{p_{2 i}^{2}(s)+p_{3 i}^{2}(s) \leqslant \mu p_{1 i-1}(s) ;} \\
& \sqrt{F_{2}^{2}\left(p_{i}, \tilde{\Delta}_{2 i}\right)_{s}+F_{3}^{2}\left(p_{i}, \tilde{\Delta}_{3 i}\right)_{s}} p_{2 i}(s)+\mu p_{1 i-1}(s) F_{2}\left(p_{i}, \tilde{\Delta}_{2 i}\right)_{s}=0 ; \\
& \sqrt{F_{2}^{2}\left(p_{i}, \tilde{\Delta}_{2 i}\right)_{s}+F_{3}^{2}\left(p_{i}, \tilde{\Delta}_{3 i}\right)_{s}} p_{3 i}(s)+\mu p_{1 i-1}(s) F_{3}\left(p_{i}, \tilde{\Delta}_{3 i}\right)_{s}=0
\end{aligned}
$$

где $s \in \Omega, i=\overline{1, l}$.

При использовании указанной модификации закона трения Кулона рассматриваемая контактная задача на $i$-том шаге нагружения сводится к определению неизвестных функций $p_{1 i}(s), p_{2 i}(s), p_{3 i}(s) \in L_{2}(\Omega)$, которые почти всюду на $\Omega$ удовлетворяют соотношениям (6). Принятую модификацию закона трения Кулона, выраженную последними тремя соотношениями системы (6), можно истолковать как наличие запаздывания в выражениях $\mu p_{1 i}(s)$, входящих в последние три соотношения системы (6) и ограничивающих модуль удельной касательной контактной нагрузки. При этом очевидно, что на $i$-том шаге нагружения ограничивающее контактное давление $p_{1 i-1}(s)$ является известным, что существенно упрощает граничные условия контактной задачи. Если система (6) решена для $i$-того шага нагружения, то конфигурацию области контакта на этом шаге можно определить следующим образом:

$$
\begin{gathered}
\Omega_{0}^{(i)}=\left\{s \in \Omega \mid F_{1}\left(p_{i}, \Delta_{1 i}\right)_{s}=0\right\}, \\
\Omega_{C}^{(i)}=\left\{s \in \Omega_{0}^{(i)} \mid F_{2}^{2}\left(p_{i}, \tilde{\Delta}_{2 i}\right)_{s}+F_{3}^{2}\left(p_{i}, \tilde{\Delta}_{3 i}\right)_{s}=0\right\}, \\
\Omega_{\Pi}^{(i)}=\left\{s \in \Omega_{0}^{(i)} \mid F_{2}^{2}\left(p_{i}, \tilde{\Delta}_{2 i}\right)_{s}+F_{3}^{2}\left(p_{i}, \tilde{\Delta}_{3 i}\right)_{s}>0\right\},
\end{gathered}
$$

где $\Omega_{0}^{(i)}$ - область контакта, $\Omega_{C}^{(i)}$ - зона сцепления, $\Omega_{\Pi}^{(i)}$ - зона проскальзывания. Отметим, что при изменении индекса $i$ от 1 до $l$ соотношения (6) представляют собой серию систем, подлежащих последовательному решению. Окончательным решением рассматриваемой квазистатической задачи условимся считать функции $p_{k l}(s), k=\overline{1,3}$ и области $\Omega_{0}^{(l)}, \Omega_{C}^{(l)}, \Omega_{\Pi}^{(l)}$, найденные на последнем шаге нагружения $l$. 
2. Операторное уравнение контактной задачи. Для неизвестных векторфункций $p_{1}(s), p_{2}(s), \ldots, p_{l}(s) \in L_{2}^{3}(\Omega)$ серия систем соотношений (6) эквивалентна серии нелинейных операторных уравнений [9]

$$
p_{i}=G_{\mu p_{1 i-1}}\left(p_{i}-E\left(A\left(p_{i}\right)-\tilde{f}_{i}\right)\right), \quad i=\overline{1, l},
$$

в которых $E$ - произвольная положительная константа, $\tilde{f}_{i} \in L_{2}^{3}(\Omega)$ и определяются равенствами

$$
\tilde{f}_{i}=\left(-\delta_{0}(s)-\Delta_{1 i},-\tilde{\Delta}_{2 i}(s),-\tilde{\Delta}_{3 i}(s)\right) .
$$

Линейный ограниченный оператор влияния $A: L_{2}^{3}(\Omega) \rightarrow L_{2}^{3}(\Omega)$, входящий в уравнения (7), задается соотношениями

$$
\begin{aligned}
& x=\left(x_{1}, x_{2}, x_{3}\right), y=\left(y_{1}, y_{2}, y_{3}\right) \in L_{2}^{3}(\Omega) ; \\
& y=A(x), y_{k}=\sum_{j=1}^{3} A_{k j}\left(x_{j}\right) \forall k=\overline{1,3} .
\end{aligned}
$$

Входящий в правую часть соотношений (7) нелинейный непрерывный оператор $G_{\mu g}: L_{2}^{3}(\Omega) \rightarrow L_{2}^{3}(\Omega)$ определяется равенствами

$$
\begin{aligned}
& x=\left(x_{1}, x_{2}, x_{3}\right), y=\left(y_{1}, y_{2}, y_{3}\right) \in L_{2}^{3}(\Omega) ; \\
& y=G_{\mu g}(x), \\
& y_{1}(s)=h\left(x_{1}(s)\right), \\
& y_{2}(s)=q\left(x_{2}(s), x_{3}(s), \mu g(s)\right), \\
& y_{3}(s)=q\left(x_{3}(s), x_{2}(s), \mu g(s)\right), s \in \Omega,
\end{aligned}
$$

в которых $g(s) \in L_{2}(\Omega)$ - заданная, почти всюду на $\Omega$ неотрицательная функция, а функции $h(x)$ и $q(x, y, z)$ определяются соотношениями

$$
\begin{aligned}
& h(x)= \begin{cases}x, & \text { если } x \geqslant 0 ; \\
0, & \text { если } x<0 ;\end{cases} \\
& q(x, y, z)=\left\{\begin{array}{cc}
x, & \text { если } \sqrt{x^{2}+y^{2}} \leqslant z ; \\
\frac{x z}{\sqrt{x^{2}+y^{2}}}, & \text { если } \sqrt{x^{2}+y^{2}}>z .
\end{array}\right.
\end{aligned}
$$

Для получения оператора $G_{\mu p_{1-1}}: L_{2}^{3}(\Omega) \rightarrow L_{2}^{3}(\Omega)$, который фактически используется в выражении правой части уравнения (7), нужно в соотношениях $(9)$, задающих оператор $G_{\mu g}: L_{2}^{3}(\Omega) \rightarrow L_{2}^{3}(\Omega)$, положить $g(s)=p_{1 i-1}(s)$ почти всюду на $\Omega$.

Важной особенностью [9] каждого из уравнений (7) является то, что множество его решений в $L_{2}^{3}(\Omega)$ не изменяется при изменении входящего в это уравнение положительного параметра $E$.

Оператор $G_{\mu g}: L_{2}^{3}(\Omega) \rightarrow L_{2}^{3}(\Omega)$, определяемый соотношениями (9), является метрическим проектором [11] гильбертова пространства $L_{2}^{3}(\Omega)$ на выпуклое замкнутое множество

$$
U=\left\{\left(x_{1}, x_{2}, x_{3}\right) \in L_{2}^{3}(\Omega) \mid x_{1}(s) \geqslant 0, \sqrt{x_{2}^{2}(s)+x_{3}^{2}(s)} \leqslant \mu g(s), s \in \Omega\right\}
$$


этого пространства. Этот факт следует из очевидных свойств функций $h(x)$ и $q(x, y, z)$, определяемых соотношениями (10). Действительно, функция $h(x)$ является метрическим проектором одномерного евклидова пространства $\mathbb{R}^{1}$ на промежуток $[0 ;+\infty) \subset \mathbb{R}^{1}$, а отображение $\varphi_{r}: \mathbb{R}^{2} \rightarrow \mathbb{R}^{2}$ вида

$$
\begin{aligned}
& x=\left(x_{1}, x_{2}\right), y=\left(y_{1}, y_{2}\right) \in \mathbb{R}^{2} \\
& y=\varphi_{r}(x) \\
& y_{1}=q\left(x_{1}, x_{2}, r\right) ; \\
& y_{2}=q\left(x_{2}, x_{1}, r\right) ; r \geqslant 0,
\end{aligned}
$$

осуществляет метрическую проекцию двумерного евклидова пространства $\mathbb{R}^{2}$ на замкнутый круг

$$
\left\{\left(x_{1}, x_{2}\right) \in \mathbb{R}^{2} \mid \sqrt{x_{1}^{2}+x_{2}^{2}} \leqslant r\right\}
$$

этого пространства. Отметим, что входящая в (7) известная неотрицательная функция $p_{1 i-1} \in L_{2}(\Omega)$ определяется из решения $p_{i-1} \in L_{2}^{3}(\Omega)$ этого уравнения на $(i-1)$-м шаге нагружения, а известный элемент $\tilde{f}_{i} \in L_{2}^{3}(\Omega)$ также зависит от решения $p_{i-1}$. Таким образом, решение $p_{i} \in L_{2}^{3}(\Omega)$ уравнения (7) при фиксированном значении индекса $i$ можно получить после нахождения решений $p_{1}, p_{2}, \ldots, p_{i-1} \in L_{2}^{3}(\Omega)$ этого уравнения на предшествующих шагах нагружения (при получении $p_{1}$ предполагается, что в момент начального касания тел вектор-функция контактной нагрузки и жесткие сближения тел тождественно равны нулю на $\Omega$ ). Окончательным решением квазистатической контактной задачи, описываемой серией уравнений (7), будем считать решение $p_{l} \in L_{2}^{3}(\Omega)$, полученное на последнем $l$-том шаге нагружения. Для многих важных для практики случаев входящие в соотношения (8) линейные ограниченные операторы $A_{k j}: L_{2}(\Omega) \rightarrow L_{2}(\Omega)$ допускают интегральное представление. В этих случаях каждое из операторных уравнений (7) при фиксированном значении индекса $i$ представляет собой систему трех нелинейных интегральных уравнений относительно трех неизвестных функций $p_{1 i}(s), p_{2 i}(s), p_{3 i}(s)$, задающих распределение удельной контактной нагрузки, передаваемой от одного тела к другому.

3. Единственность решения контактной задачи. Для доказательства единственности решения контактной задачи, описываемой серией операторных уравнений (7), достаточно доказать единственность решения каждого из уравнений этой серии. Фиксируя индекс $i$ в (7) и обозначая $p=p_{i} \in L_{2}^{3}(\Omega)$, $g=p_{1 i-1} \in L_{2}(\Omega), b=\tilde{f}_{i} \in L_{2}^{3}(\Omega)$, получим более простой вид каждого операторного уравнения из совокупности (7):

$$
p=G_{\mu g}(p-E(A(p)-b)) .
$$

В дальнейшем будем полагать, что линейный ограниченный оператор влияния $A: L_{2}^{3}(\Omega) \rightarrow L_{2}^{3}(\Omega)$, входящий в уравнение $(11)$, является самосопряженным и положительным:

$$
\begin{aligned}
& (A(x), y)=(x, A(y)) \forall x, y \in L_{2}^{3}(\Omega), \\
& (A(x), x)>0 \quad \forall x \in L_{2}^{3}(\Omega) \backslash\left\{\theta_{L_{2}^{3}(\Omega)}\right\},
\end{aligned}
$$


где $(x, y)$-скалярное произведение элементов $x, y$ пространства $L_{2}^{3}(\Omega)$, $\theta_{L_{2}^{3}(\Omega)}$ - нулевой элемент пространства $L_{2}^{3}(\Omega)$. Самосопряженность оператора $A(p)$ обусловлена свойством взаимности упругой среды, а его положительность гарантирована положительностью энергии упругих деформаций системы взаимодействующих тел, подверженных ненулевому нагружению.

Сформулируем и докажем теорему о единственности решения уравнения (11) при условии, что оператор влияния $A$ удовлетворяет соотношениям (12).

Tеорема 1. Пусть линейный ограниченный оператор $A: L_{2}^{3}(\Omega) \rightarrow L_{2}^{3}(\Omega)$ удовлетворяет условиям (12). Тогда для любого элемента $b \in L_{2}^{3}(\Omega)$ любой заданной и почти всюду неотрицательной на $\Omega$ функиии $g \in L_{2}(\Omega)$ и любого действительного числа $\mu>0$ уравнение (11) не может иметь более одного решения в пространстве $L_{2}^{3}(\Omega)$.

Доказ ательство. Предположим противное. Пусть $p$ и $\tilde{p}$ - два различных решения уравнения (11) в пространстве $L_{2}^{3}(\Omega)$. Для оценивания нормы разности элементов $p$ и $\tilde{p}$ воспользуемся нерастягиваемостью на этом пространстве метрического проектора $G_{\mu g}: L_{2}^{3}(\Omega) \rightarrow L_{2}^{3}(\Omega)[11]:$

$$
\begin{aligned}
\|p-\tilde{p}\|=\left\|G_{\mu g}(p-E(A(p)-b))-G_{\mu g}(\tilde{p}-E(A(\tilde{p})-b))\right\| \leqslant & \\
& \leqslant\|p-\tilde{p}-E A(p-\tilde{p})\| .
\end{aligned}
$$

Возведя обе части полученного неравенства в квадрат и перейдя в нём от норм элементов к скалярным произведениям, получим оценку

$$
\|p-\tilde{p}\|^{2} \leqslant\|p-\tilde{p}\|^{2}-2 E(p-\tilde{p}, A(p-\tilde{p}))+E^{2}\|A(p-\tilde{p})\|^{2},
$$

означающую справедливость неравенства

$$
(p-\tilde{p}, A(p-\tilde{p})) \leqslant \frac{E}{2}\|A(p-\tilde{p})\|^{2} .
$$

Очевидно, что полученное неравенство будет выполняться для любого положительного значения $E$. Переходя в этом неравенстве к пределу при $E \rightarrow+0$, получим соотношение

$$
(p-\tilde{p}, A(p-\tilde{p})) \leqslant 0,
$$

которое противоречит второму из условий в (12). Полученное противоречие означает, что доказываемое утверждение верно.

Доказанная теорема означает, что при выполнении её условий рассматриваемая квазистатическая контактная задача, описываемая серией уравнений $(7)$, не может иметь более одного решения в пространстве $L_{2}^{3}(\Omega)$.

4. Регуляризация операторного уравнения контактной задачи. Разработка численного алгоритма решения уравнения (11) осложняется тем, что во многих случаях входящий в него линейный оператор влияния $A: L_{2}^{3}(\Omega) \rightarrow L_{2}^{3}(\Omega)$ является вполне непрерывным $[9,11]$. Это обстоятельство приводит к тому, что задача нахождения неизвестного элемента $p \in L_{2}^{3}(\Omega)$ по известным элементам $b \in L_{2}^{3}(\Omega), g \in U_{0}$ из решения уравнения (11) является некорректно поставленной в смысле А. Н. Тихонова [12] (здесь и ниже символом $U_{0}$ обозначен конус неотрицательных функций пространства $L_{2}(\Omega)$ [13]). Поэтому 
вместо уравнения (11) целесообразно рассматривать его регуляризированный аналог

$$
p=G_{\mu g}(p-E(\varepsilon p+A(p)-b)),
$$

в котором $\varepsilon>0$ есть некоторое фиксированное число - параметр регуляризации. Сформулируем и докажем две теоремы, из которых следует, что уравнение (13) является регуляризированным аналогом уравнения (11).

Теорема 2. Пусть линейный ограниченный оператор $A: L_{2}^{3}(\Omega) \rightarrow L_{2}^{3}(\Omega)$ удовлетворяет условиям (12). Тогда для любого фиксированного положительного числа в задача нахождения неизвестного элемента $p \in L_{2}^{3}(\Omega)$ по известным элементам $b \in L_{2}^{3}(\Omega), g \in U_{0}$ из решения уравнения (13) является корректно поставленной в смысле А. Н. Тихонова.

Доказ а т ель ство. Для доказательства теоремы достаточно показать, что для любых элементов $b \in L_{2}^{3}(\Omega), g \in U_{0}$ уравнение (13) однозначно разрешимо в пространстве $L_{2}^{3}(\Omega)$ и его решение $p \in L_{2}^{3}(\Omega)$ непрерывно зависит от элементов $b \in L_{2}^{3}(\Omega), g \in U_{0}[12]$.

Пусть $\varepsilon-$ фиксированное положительное число и $b \in L_{2}^{3}(\Omega), g \in U_{0}$. Выберем положительное число $E$ в уравнении (13) так, чтобы выполнялось неравенство

$$
E<\frac{1}{\varepsilon+\|A\|_{*}},
$$

где $\|A\|_{*}$ - норма оператора $A: L_{2}^{3}(\Omega) \rightarrow L_{2}^{3}(\Omega)[14]$.

Докажем, что из неравенства (14) следует оценка

$$
\|I-E(\varepsilon I+A)\|_{*} \leqslant 1-E \varepsilon<1,
$$

где $I$ - тожественный оператор, действующий в пространстве $L_{2}^{3}(\Omega)$. Из неравенства (14) и условий (12) следует, что линейный ограниченный оператор $(I-E(\varepsilon I+A)): L_{2}^{3}(\Omega) \rightarrow L_{2}^{3}(\Omega)$ является самосопряженным и положительным. Найдя его норму с помощью известного соотношения [14], получим для этой нормы очевидную оценку:

$$
\begin{aligned}
\|I-E(\varepsilon I+A)\|_{*}=\sup _{\|x\|=1}( & x-E(\varepsilon x+A(x)), x)= \\
& =1-E \varepsilon-E \inf _{\|x\|=1}(A(x), x) \leqslant 1-E \varepsilon=q<1,
\end{aligned}
$$

из которой следует неравенство (15). Из этого неравенства и нерастягиваемости метрического проектора $G_{\mu g}: L_{2}^{3}(\Omega) \rightarrow L_{2}^{3}(\Omega)$ следует сжимаемость оператора $F: L_{2}^{3}(\Omega) \rightarrow L_{2}^{3}(\Omega)$, стоящего в правой части уравнения (13). Действительно, для любых элементов $x, y \in L_{2}^{3}(\Omega)$ можно получить оценки

$$
\begin{aligned}
\|F(x)-F(y)\|= & \\
=\| G_{\mu g}(x- & E(\varepsilon x+A(x)-b))-G_{\mu g}(y-E(\varepsilon y+A(y)-b)) \| \leqslant \\
& \leqslant\|x-y-E(\varepsilon(x-y)+A(x-y))\| \leqslant \\
& \leqslant\|I-E(\varepsilon I+A)\|_{*} \cdot\|x-y\| \leqslant q\|x-y\|,
\end{aligned}
$$


где $0<q<1$. Таким образом, однозначная разрешимость уравнения (13) в пространстве $L_{2}^{3}(\Omega)$ для любых элементов $b \in L_{2}^{3}(\Omega), g \in U_{0}$ следует из принципа сжимающих отображений [15].

Пусть элементы $p^{\prime}, p^{\prime \prime} \in L_{2}^{3}(\Omega)$ являются решениями уравнения (13), полученными при $b=b^{\prime} \in L_{2}^{3}(\Omega), g=g^{\prime} \in U_{0}$ и $b=b^{\prime \prime} \in L_{2}^{3}(\Omega), g=g^{\prime \prime} \in U_{0}$ соответственно. Тогда, учитывая нерастягиваемость оператора $G_{\mu g}: L_{2}^{3}(\Omega) \rightarrow L_{2}^{3}(\Omega)$ и соотношения (10), (15), можно получить неравенства

$$
\begin{aligned}
& \left\|p^{\prime}-p^{\prime \prime}\right\|= \\
& =\left\|G_{\mu g^{\prime}}\left(p^{\prime}-E\left(\varepsilon p^{\prime}+A\left(p^{\prime}\right)-b^{\prime}\right)\right)-G_{\mu g^{\prime \prime}}\left(p^{\prime \prime}-E\left(\varepsilon p^{\prime \prime}+A\left(p^{\prime \prime}\right)-b^{\prime \prime}\right)\right)\right\|= \\
& =\| G_{\mu g^{\prime}}\left(p^{\prime}-E\left(\varepsilon p^{\prime}+A\left(p^{\prime}\right)-b^{\prime}\right)\right)-G_{\mu g^{\prime}}\left(p^{\prime \prime}-E\left(\varepsilon p^{\prime \prime}+A\left(p^{\prime \prime}\right)-b^{\prime \prime}\right)\right)+ \\
& +G_{\mu g^{\prime}}\left(p^{\prime \prime}-E\left(\varepsilon p^{\prime \prime}+A\left(p^{\prime \prime}\right)-b^{\prime \prime}\right)\right)-G_{\mu g^{\prime \prime}}\left(p^{\prime \prime}-E\left(\varepsilon p^{\prime \prime}+A\left(p^{\prime \prime}\right)-b^{\prime \prime}\right)\right) \| \leqslant \\
& \leqslant\left\|G_{\mu g^{\prime}}\left(p^{\prime}-E\left(\varepsilon p^{\prime}+A\left(p^{\prime}\right)-b^{\prime}\right)\right)-G_{\mu g^{\prime}}\left(p^{\prime \prime}-E\left(\varepsilon p^{\prime \prime}+A\left(p^{\prime \prime}\right)-b^{\prime \prime}\right)\right)\right\|+ \\
& +\left\|G_{\mu g^{\prime}}\left(p^{\prime \prime}-E\left(\varepsilon p^{\prime \prime}+A\left(p^{\prime \prime}\right)-b^{\prime \prime}\right)\right)-G_{\mu g^{\prime \prime}}\left(p^{\prime \prime}-E\left(\varepsilon p^{\prime \prime}+A\left(p^{\prime \prime}\right)-b^{\prime \prime}\right)\right)\right\| \leqslant \\
& \leqslant\left\|\left(p^{\prime}-p^{\prime \prime}\right)-E\left(\varepsilon\left(p^{\prime}-p^{\prime \prime}\right)+A\left(p^{\prime}-p^{\prime \prime}\right)\right)+E\left(b^{\prime}-b^{\prime \prime}\right)\right\|+\mu\left\|g^{\prime}-g^{\prime \prime}\right\|_{1} \leqslant \\
& \leqslant\|I-E(\varepsilon I+A)\|_{*} \cdot\left\|p^{\prime}-p^{\prime \prime}\right\|+E\left\|b^{\prime}-b^{\prime \prime}\right\|+\mu\left\|g^{\prime}-g^{\prime \prime}\right\|_{1} \leqslant \\
& \leqslant(1-E \varepsilon)\left\|p^{\prime}-p^{\prime \prime}\right\|+E\left\|b^{\prime}-b^{\prime \prime}\right\|+\mu\left\|g^{\prime}-g^{\prime \prime}\right\|_{1},
\end{aligned}
$$

из которых для нормы $\left\|p^{\prime}-p^{\prime \prime}\right\|$ следует оценка

$$
\left\|p^{\prime}-p^{\prime \prime}\right\| \leqslant \frac{1}{\varepsilon}\left\|b^{\prime}-b^{\prime \prime}\right\|+\frac{\mu}{E \varepsilon}\left\|g^{\prime}-g^{\prime \prime}\right\|_{1} .
$$

Здесь и ниже символами $\|\cdot\|$ и $\|\cdot\|_{1}$ обозначены нормы элементов пространств $L_{2}^{3}(\Omega)$ и $L_{2}(\Omega)$ соответственно. Эта оценка, полученная при фиксированном $\varepsilon>0$ для любых элементов $b^{\prime}, b^{\prime \prime} \in L_{2}^{3}(\Omega)$ и $g^{\prime}, g^{\prime \prime} \in U_{0}$, означает, что решение уравнения $(13)$ в пространстве $L_{2}^{3}(\Omega)$ непрерывно зависит от элементов $b \in L_{2}^{3}(\Omega), g \in U_{0}$.

Теорема 3. Пусть линейный вполне непреръвный оператор $A: L_{2}^{3}(\Omega) \rightarrow$ $L_{2}^{3}(\Omega)$ удовлетворяет условиям (12) и для некоторых биксированных элементов $b \in L_{2}^{3}(\Omega), g \in U_{0}$ уравнение $(11)$ имеет решение $p^{*} \in L_{2}^{3}(\Omega)$. Тогда

$$
\lim _{\varepsilon \rightarrow+0}\left\|p^{*}-p_{\varepsilon}\right\|=0
$$

где $p_{\varepsilon} \in L_{2}^{3}(\Omega)$ является решением уравнения (13), полученнъм при заданном положительном значении параметра регуляризачии в.

Доказ ательств о. Из очевидных равенств

$$
p^{*}=G_{\mu g}\left(p^{*}-E\left(A\left(p^{*}\right)-b\right)\right), \quad p_{\varepsilon}=G_{\mu g}\left(p_{\varepsilon}-E\left(\varepsilon p_{\varepsilon}+A\left(p_{\varepsilon}\right)-b\right)\right)
$$

вытекает оценка

$$
\left\|p^{*}-p_{\varepsilon}\right\|^{2} \leqslant\left\|p^{*}-p_{\varepsilon}-E\left(A\left(p^{*}-p_{\varepsilon}\right)-\varepsilon p_{\varepsilon}\right)\right\|^{2},
$$

справедливая для любого числа $E>0$. Переходя в этой оценке от норм элементов к скалярным произведениям, получим неравенство

$$
\left(p^{*}-p_{\varepsilon}, A\left(p^{*}-p_{\varepsilon}\right)-\varepsilon p_{\varepsilon}\right) \leqslant \frac{E}{2}\left\|A\left(p^{*}-p_{\varepsilon}\right)-\varepsilon p_{\varepsilon}\right\|^{2} .
$$


Переходя в этом неравенстве к пределу при $E \rightarrow+0$, получим условие

$$
\left(p^{*}-p_{\varepsilon}, A\left(p^{*}-p_{\varepsilon}\right)-\varepsilon p_{\varepsilon}\right) \leqslant 0 \text {. }
$$

Используя положительность оператора $A$ и неравенство Коши-Буняковского [15], получим из условия (16) оценки

$$
0 \leqslant\left(p^{*}-p_{\varepsilon}, A\left(p^{*}-p_{\varepsilon}\right)\right) \leqslant\left(p^{*}-p_{\varepsilon}, \varepsilon p_{\varepsilon}\right) \leqslant \varepsilon\left\|p_{\varepsilon}\right\|\left(\left\|p^{*}\right\|-\left\|p_{\varepsilon}\right\|\right),
$$

влекущие за собой неравенство

$$
\left\|p_{\varepsilon}\right\| \leqslant\left\|p^{*}\right\|
$$

Очевидно, что неравенства (16) и (17) выполняются для любого числа $\varepsilon>0$.

Предположим теперь, что $\left\{\varepsilon_{n}\right\}$ - произвольная последовательность положительных чисел, для которой

$$
\lim _{n \rightarrow \infty} \varepsilon_{n}=0
$$

Введя обозначение $p_{\varepsilon_{n}}=p_{n}$, покажем, что

$$
\lim _{n \rightarrow \infty}\left\|p_{n}-p^{*}\right\|=0
$$

Это и будет означать справедливость доказываемой теоремы.

Для доказательства сильной сходимости последовательности $\left\{p_{n}\right\}$ элементов $L_{2}^{3}(\Omega)$ к элементу $p^{*} \in L_{2}^{3}(\Omega)$ достаточно показать, что из любой подпоследовательности $\left\{p_{n_{k}}\right\}$ последовательности $\left\{p_{n}\right\}$ можно извлечь другую подпоследовательность $\left\{p_{n_{k_{j}}}\right\}$, которая сильно сходится к элементу $p^{*}$. Покажем это.

Пусть $\left\{p_{n_{k}}\right\}$ - произвольная подпоследовательность последовательности $\left\{p_{n}\right\}$. Поскольку подпоследовательность $\left\{p_{n_{k}}\right\}$ является ограниченной в силу неравенства (17), существует такая её подпоследовательность $\left\{p_{n_{k_{j}}}\right\}$ и такой элемент $\tilde{p} \in L_{2}^{3}(\Omega)$, что $\left\{p_{n_{k_{j}}}\right\}$ слабо сходится в $L_{2}^{3}(\Omega)$ к $\tilde{p}$ (см. $[11$, теорема 1.7]). Из оценки (16) при $\varepsilon=\varepsilon_{n_{k_{j}}}$ вытекает неравенство

$$
\left(p^{*}-p_{n_{k_{j}}}, A\left(p^{*}-p_{n_{k_{j}}}\right)\right) \leqslant \varepsilon_{n_{k_{j}}}\left(p^{*}, p_{n_{k_{j}}}\right)-\varepsilon_{n_{k_{j}}}\left\|p_{n_{k_{j}}}\right\|^{2}
$$

справедливое для всех значений индекса $n_{k_{j}}$. Переходя в этом неравенстве к пределу при $n_{k_{j}} \rightarrow \infty$, получим неравенство

$$
\left(p^{*}-\tilde{p}, A\left(p^{*}-\tilde{p}\right)\right) \leqslant 0,
$$

которое в силу второго из условий $(12)$ означает, что $p^{*}=\tilde{p}$. Следовательно, $\left\{p_{n_{k_{j}}}\right\}$ слабо сходится к элементу $p^{*}$. Но поскольку ещё из неравенства (17) следуют оценки

$$
\left\|p_{n_{k_{j}}}\right\| \leqslant\left\|p^{*}\right\| \forall n_{k_{j}}
$$

подпоследовательность $\left\{p_{n_{k_{j}}}\right\}$ сильно сходится к $p^{*}$ (см. [11, теорема 1.4]). 
Из доказанных теорем 2 и 3 следует, что уравнение (13) является регуляризирующим аналогом уравнения (11).

5. Дискретизация регуляризированного уравнения. Наличие слагаемого $\varepsilon p$ в уравнении (13) позволяет построить эффективный устойчивый численный алгоритм приближенного решения этого уравнения. Такой алгоритм основывается на следующей аппроксимационной теореме.

ТЕОрема 4. Пустъ $b \in L_{2}^{3}(\Omega), g \in U_{0}$, a линейный ограниченный оператор $A: L_{2}^{3}(\Omega) \rightarrow L_{2}^{3}(\Omega)$ удовлетворяет условиям (12). Пусть последовательность $\left\{A_{n}\right\}$ линейных ограниченных операторов, действующих в $L_{2}^{3}(\Omega)$, а также последовательность $\left\{b_{n}\right\}$ элементов $L_{2}^{3}(\Omega)$ и последовательность $\left\{g_{n}\right\}$ элементов $U_{0}$ удовлетворяют следующим условиям:

$$
\begin{aligned}
& \lim _{n \rightarrow \infty}\left\|A-A_{n}\right\|_{*}=0, \\
& \lim _{n \rightarrow \infty}\left\|b-b_{n}\right\|=0, \\
& \lim _{n \rightarrow \infty}\left\|g-g_{n}\right\|_{1}=0 .
\end{aligned}
$$

Тогда для любого числа $\varepsilon>0$ существует натуральное число $n_{0}$, зависящее только от $\varepsilon$, такое, что для всех натуральных $n \geqslant n_{0}$ уравнение

$$
p=G_{\mu g_{n}}\left(p-E\left(\varepsilon p+A_{n}(p)-b_{n}\right)\right)
$$

является однозначно разрешимым в $L_{2}^{3}(\Omega)$ и справедливо равенство

$$
\lim _{n \rightarrow \infty}\left\|p_{n}-p_{\varepsilon}\right\|=0
$$

где $p_{n}, p_{\varepsilon} \in L_{2}^{3}(\Omega)$ являются решениями уравнений (19) и (13) соответственно.

Д о каз а т ел в с $m$ в о. Выберем для заданного числа $\varepsilon>0$ номер $n_{0}$ и число $E>0$ в уравнении (19) так, чтобы выполнялись следующие неравенства:

$$
\left\|A-A_{n}\right\|_{*}<\frac{\varepsilon}{2} \forall n \geqslant n_{0}, \quad E<\frac{1}{\varepsilon+\|A\|_{*}} .
$$

Используя эти неравенства, можно получить оценку

$$
\left\|I-E\left(\varepsilon I+A_{n}\right)\right\|_{*} \leqslant 1-E \frac{\varepsilon}{2}<1 \quad \forall n \geqslant n_{0} .
$$

Действительно,

$$
\begin{gathered}
\left\|I-E\left(\varepsilon I+A_{n}\right)\right\|_{*}=\left\|I-E\left(\varepsilon I+A_{n}-A+A\right)\right\|_{*} \leqslant \\
\leqslant\|I-E(\varepsilon I+A)\|_{*}+E\left\|A-A_{n}\right\|_{*} \leqslant \sup _{\|x\|=1}(x-E(\varepsilon x+A(x)), x)+E \frac{\varepsilon}{2} \leqslant \\
\leqslant 1-E \varepsilon+E \frac{\varepsilon}{2}=1-E \frac{\varepsilon}{2}<1 .
\end{gathered}
$$

Из неравенства (20) следует сжимаемость оператора $F_{n}: L_{2}^{3}(\Omega) \rightarrow L_{2}^{3}(\Omega)$, стоящего в правой части уравнения (19) (см. доказательство теоремы 2), которая 
влечет за собой однозначную разрешимость этого уравнения в пространстве $L_{2}^{3}(\Omega)$ для всех натуральных $n \geqslant n_{0}$.

Докажем теперь сильную сходимость в пространстве $L_{2}^{3}(\Omega)$ последовательности $\left\{p_{n}\right\}$ решений уравнений $(19)$ к решению $p_{\varepsilon} \in L_{2}^{3}(\Omega)$ уравнения (13). Для этого рассмотрим последовательность $\left\{\alpha_{n}\right\}$ элементов $L_{2}^{3}(\Omega)$, определяемую равенством

$$
\alpha_{n}=A_{n}\left(p_{n}\right)-A\left(p_{n}\right)+b-b_{n}, \quad n \geqslant n_{0} .
$$

Учитывая условия (18) и ограниченность в $L_{2}^{3}(\Omega)$ последовательности $\left\{p_{n}\right\}$, имеющую место в силу ограниченности в $L_{2}^{3}(\Omega)$ сходящейся последовательности $\left\{b_{n}\right\}$, а также неравенства $(20)$, получим для $\alpha_{n}$ оценки

$$
\left\|\alpha_{n}\right\| \leqslant\left\|A_{n}-A\right\|_{*}\left\|p_{n}\right\|+\left\|b-b_{n}\right\| \leqslant\left\|A_{n}-A\right\|_{*} \cdot \tilde{\beta}+\left\|b-b_{n}\right\|,
$$

где $\tilde{\beta}$ - некоторое положительное число. Из последних оценок следует, что

$$
\lim _{n \rightarrow \infty}\left\|\alpha_{n}\right\|=0
$$

Поскольку из теоремы 2 следует непрерывная зависимость решения уравнения (13) от элементов $b \in L_{2}^{3}(\Omega), g \in U_{0}$, из очевидных соотношений

$$
\begin{gathered}
p_{\varepsilon}=G_{\mu g}\left(p_{\varepsilon}-E\left(\varepsilon p_{\varepsilon}+A\left(p_{\varepsilon}\right)-b\right)\right), \\
p_{n}=G_{\mu g_{n}}\left(p_{n}-E\left(\varepsilon p_{n}+A\left(p_{n}\right)-b+\alpha_{n}\right)\right),
\end{gathered}
$$

а также из последнего равенства (18) и доказанного соотношения (21) следует, что $\lim _{n \rightarrow \infty}\left\|p_{n}-p_{\varepsilon}\right\|=0$.

Из теорем 1-4 следует, что приближенное решение рассматриваемой контактной задачи на каждом шаге нагружения можно получить, решив уравнение (19) при достаточно малом значении $\varepsilon>0$ и достаточной близости оператора $A_{n}$ и элементов $b_{n}, g_{n}$ к оператору $A$ и элементам $b, g$ соответственно.

Построим такие удовлетворяющие условиям теоремы 4, аппроксимирующие последовательности $\left\{A_{n}\right\},\left\{b_{n}\right\}$ и $\left\{g_{n}\right\}$, для которых уравнение (19) можно решить численно с любой степенью точности. Для этого зададим область $\Omega$ в виде открытого квадрата, ограниченного прямыми, параллельными осям некоторой декартовой системы координат, введенной на общей для взаимодействующих тел касательной плоскости. Далее для каждого натурального $n$ разобьем область $\Omega$ на $n^{2}$ непересекающихся квадратных областей $\omega_{1}, \omega_{2}, \ldots, \omega_{n^{2}}$ равной площади, ориентированных подобно квадрату $\Omega$.

Будем полагать, что в выражениях (8) линейные ограниченные операторы $A_{i j}: L_{2}(\Omega) \rightarrow L_{2}(\Omega)$ допускают интегральное представление

$$
A_{i j}(x)_{t}=\int_{\Omega} K_{i j}(t, s) x(s) d s, \quad t \in \Omega, \quad i, j=\overline{1,3}
$$

и являются вполне непрерывными. Зададим линейный вполне непрерывный оператор $A_{n}: L_{2}^{3}(\Omega) \rightarrow L_{2}^{3}(\Omega)$ следующим образом: 


$$
\begin{aligned}
& x=\left(x_{1}, x_{2}, x_{3}\right), y=\left(y_{1}, y_{2}, y_{3}\right) \in L_{2}^{3}(\Omega) \\
& y=A_{n}(x), y_{i}=\sum_{j=1}^{3} A_{i j}^{(n)}\left(x_{j}\right), i=\overline{1,3} \\
& A_{i j}^{(n)}\left(x_{j}\right)_{t}=\int_{\Omega} K_{i j}^{(n)}(t, s) x_{j}(s) d s, t \in \Omega, i, j=\overline{1,3}
\end{aligned}
$$

Входящие в соотношения $(22)$ функции $K_{i j}^{(n)}(t, s)$ определяются равенствами

$$
\begin{aligned}
& K_{i j}^{(n)}(t, s)=\frac{1}{\operatorname{mes}\left(\omega_{k}\right) \operatorname{mes}\left(\omega_{m}\right)} \int_{\omega_{k}}\left(\int_{\omega_{m}} K_{i j}(t, s) d s\right) d t, \\
& \text { если } t \in \omega_{k}, s \in \omega_{m} ; k, m=\overline{1, n^{2}} ; i, j=\overline{1,3},
\end{aligned}
$$

в которых $\operatorname{mes}\left(\omega_{k}\right)$ есть площадь квадрата $\omega_{k}$. Элементы $b_{n} \in L_{2}^{3}(\Omega)$ и $g_{n} \in U_{0}$ зададим в виде $b_{n}=\left(P_{n}\left(\tilde{b}_{1}\right), P_{n}\left(\tilde{b}_{2}\right), P_{n}\left(\tilde{b}_{3}\right)\right), g_{n}=P_{n}(g)$, где $\left(\tilde{b}_{1}, \tilde{b}_{2}, \tilde{b}_{3}\right)=b$ и оператор $P_{n}: L_{2}(\Omega) \rightarrow L_{2}(\Omega)$ определяется соотношениями

$$
P_{n}(x)_{t}=\frac{1}{\operatorname{mes}\left(\omega_{k}\right)} \int_{\omega_{k}} x(s) d s, \quad t \in \omega_{k}, k=\overline{1, n^{2}}
$$

Очевидно, что в случае полной непрерывности линейных интегральных операторов $A_{i j}: L_{2}(\Omega) \rightarrow L_{2}(\Omega)$ построенные аппроксимирующие последовательности $\left\{A_{n}\right\},\left\{b_{n}\right\}$ и $\left\{g_{n}\right\}$ удовлетворяют условиям (18). Кроме этого, эти последовательности заданы так, что оператор $F_{n}: L_{2}^{3}(\Omega) \rightarrow L_{2}^{3}(\Omega)$, стоящий в правой части уравнения (19), отображает пространство $L_{2}^{3}(\Omega)$ в конечномерное подпространство $H_{n}=P_{n}\left(L_{2}(\Omega)\right) \times P_{n}\left(L_{2}(\Omega)\right) \times P_{n}\left(L_{2}(\Omega)\right)$ пространства $L_{2}^{3}(\Omega)$. Следовательно, каждое решение $p_{n} \in L_{2}^{3}(\Omega)$ уравнения (19) содержится в $H_{n}$ и может быть найдено из решения следующей системы $3 n^{2}$ скалярных уравнений с $3 n^{2}$ неизвестными:

$$
\begin{aligned}
& x_{3 k-2}=h\left(x_{3 k-2}-E\left(\sum_{j=1}^{3 n^{2}} a_{3 k-2 j} x_{j}-b_{3 k-2}^{\prime}\right)\right) \\
& x_{3 k-1}=q\left(x_{3 k-1}-E\left(\sum_{j=1}^{3 n^{2}} a_{3 k-1 j} x_{j}-b_{3 k-1}^{\prime}\right),\right. \\
& x_{3 k}=q\left(x_{3 k}-E\left(\sum_{j=1}^{3 n^{2}} a_{3 k j} x_{j}-b_{3 k}^{\prime}\right),\right. \\
& \left.x_{3 k}-E\left(\sum_{j=1}^{3 n^{2}} a_{3 k j} x_{j}-b_{3 k}^{\prime}\right), \mu g_{k}\right) ; \\
& \left.x_{3 k-1}-E\left(\sum_{j=1}^{3 n^{2}} a_{3 k-1 j} x_{j}-b_{3 k-1}^{\prime}\right), \mu g_{k}\right)
\end{aligned}
$$


Решение $p_{n}=\left(p_{1 n}, p_{2 n}, p_{3 n}\right) \in H_{n}$ уравнения (19) выражается через решение $x_{1}, x_{2}, \ldots, x_{3 n^{2}}$ системы $(24)$ соотношениями $x_{3 k-2}=p_{1 n}(s), x_{3 k-1}=p_{2 n}(s)$, $x_{3 k}=p_{3 n}(s)$ при $s \in \omega_{k} \forall k=\overline{1, n^{2}}$. Неотрицательные постоянные $g_{k}$ в этой системе являются известными, а $\mu$-коэффициент трения. Значения параметров $b_{i}^{\prime}, a_{i j}, g_{k}$ в (24) с учетом $(22)$, (23) определяются следующими соотношениями:

$$
\begin{aligned}
& b_{3 k-2}^{\prime}=\frac{1}{\operatorname{mes}\left(\omega_{k}\right)} \int_{\omega_{k}} \tilde{b}_{1}(s) d s, \quad b_{3 k-1}^{\prime}=\frac{1}{\operatorname{mes}\left(\omega_{k}\right)} \int_{\omega_{k}} \tilde{b}_{2}(s) d s,
\end{aligned}
$$

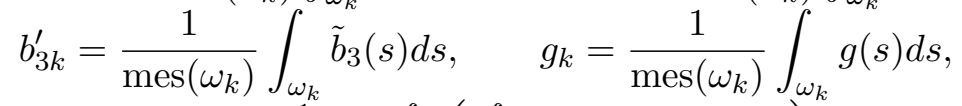

$$
\begin{aligned}
& a_{3 k-d 3 j-e}=\frac{1}{\operatorname{mes}\left(\omega_{k}\right)} \int_{\omega_{k}}\left(\int_{\omega_{j}} K_{3-d 3-e}(t, s) d s\right) d t+\varepsilon \delta_{3 k-d 3 j-e}, \\
& k, j=\overline{1, n^{2}}, d, e=\overline{0,2} \text {. }
\end{aligned}
$$

Здесь $b=\left(\tilde{b}_{1}, \tilde{b}_{2}, \tilde{b}_{3}\right), \delta_{i j}$ - символ Кронекера, а положительное число $\varepsilon-$ параметр регуляризации. Предложенный способ построения аппроксимирующих последовательностей $\left\{A_{n}\right\},\left\{b_{n}\right\},\left\{g_{n}\right\}$ гарантирует, что решение $p_{n} \in$ $L_{2}^{3}(\Omega)$ операторного уравнения (19) (существование и единственность которого в $L_{2}^{3}(\Omega)$ при достаточно большом $n$ вытекает из теоремы 4) непременно должно содержаться в конечномерном подпространстве $H_{n}$ пространства $L_{2}^{3}(\Omega)$. Это означает, что при выполнении всех условий теоремы 4 (а также при полной непрерывности линейных интегральных операторов $A_{i j}: L_{2}(\Omega) \rightarrow$ $\left.L_{2}(\Omega)\right)$ система уравнений $(24)$ является совместной, если только значение $n$ достаточно велико. Таким образом, искомое решение $p_{n}$ уравнения (19) при достаточно большом $n$ может быть восстановлено по найденным значениям неизвестных $x_{1}, x_{2}, \ldots, x_{3 n^{2}}$, удовлетворяющих системе уравнений $(24)$.

Для многих контактных задач теории упругости используется аппроксимация взаимодействующих тел упругими полупространствами [10]. В этом случае для последовательности $\left\{A_{n}\right\}$, определяемой формулами $(22),(23)$, можно вместо формул (23) использовать следующие соотношения [9]:

$$
K_{d e}^{(n)}(t, s)=\left\{\begin{array}{l}
K_{d e}\left(s_{i}^{n}, s_{j}^{n}\right), \text { если } t \in \omega_{i}, s \in \omega_{j}, i \neq j ; \\
\frac{1}{\operatorname{mes}\left(\omega_{i}\right)} \int_{\omega_{i}} K_{d e}\left(s_{i}^{n}, s\right) d s, \text { если } t, s \in \omega_{i},
\end{array}\right.
$$

где $i, j=\overline{1, n^{2}}, d, e=\overline{1,3}$. В соотношениях $(26) s_{i}^{n}$ - центр квадрата $\omega_{i}$, а ядра $K_{i j}(t, s)$ интегральных операторов $A_{i j}$ определяются в соответствии с формулами Буссинеска-Черрути [10].

Таким образом, в случае аппроксимации взаимодействующих тел упругими полупространствами коэффициенты $a_{i j}$ в системе $(24)$ можно вычислить без использования соотношений (25) по следующим простым формулам:

$$
\begin{aligned}
& a_{3 k-23 k-1}=a_{3 k-23 k}=a_{3 k-13 k-2}=a_{3 k-13 k}=a_{3 k 3 k-2}=a_{3 k 3 k-1}=0, \\
& a_{3 k-d 3 j-e}=\operatorname{mes}\left(\omega_{j}\right) K_{3-d 3-e}\left(s_{k}^{n}, s_{j}^{n}\right), \\
& a_{3 k-d 3 k-d}=\varepsilon+\int_{\omega_{k}} K_{3-j 3-j}\left(s_{k}^{n}, s\right) d s, \\
& \forall k, j=\overline{1, n^{2}} \quad(k \neq j), \quad \forall d, e=\overline{0,2} .
\end{aligned}
$$


Отметим, что при аппроксимации взаимодействующих тел упругими полупространствами линейные интегральные операторы $A_{i j}: L_{2}(\Omega) \rightarrow L_{2}(\Omega)$ являются вполне непрерывными [15], а порождаемый ими вполне непрерывный оператор $A: L_{2}^{3}(\Omega) \rightarrow L_{2}^{3}(\Omega)$ вида (8) удовлетворяет всем условиям теорем $1-4$.

6. Итерационный процесс для решения дискретного аналога регуляризированного уравнения. Из всех доказанных выше утверждений следует, что нахождение приближенного решения операторного уравнения (11), соответствующего определенному шагу нагружения рассматриваемой квазистатической контактной задачи, сводится к решению системы нелинейных скалярных уравнений (24) при достаточно малом $\varepsilon>0$ и достаточно большом натуральном $n$. Для приближенного решения системы (24) можно предложить следующий итерационный процесс:

$$
\begin{aligned}
& \left(x_{1}^{(0)}, x_{2}^{(0)}, \ldots, x_{3 n^{2}}^{(0)}\right) \in \mathbb{R}^{3 n^{2}} ; \\
& x_{3 k-2}^{(m+1)}=h\left(x_{3 k-2}^{(m)}-E\left(\sum_{j=1}^{3 n^{2}} a_{3 k-2 j} x_{j}^{(m)}-b_{3 k-2}^{\prime}\right)\right) ; \\
& x_{3 k-1}^{(m+1)}=q\left(x_{3 k-1}^{(m)}-E\left(\sum_{j=1}^{3 n^{2}} a_{3 k-1 j} x_{j}^{(m)}-b_{3 k-1}^{\prime}\right),\right. \\
& \left.x_{3 k}^{(m)}-E\left(\sum_{j=1}^{3 n^{2}} a_{3 k j} x_{j}^{(m)}-b_{3 k}^{\prime}\right), \mu g_{k}\right) ; \\
& x_{3 k}^{(m+1)}=q\left(x_{3 k}^{(m)}-E\left(\sum_{j=1}^{3 n^{2}} a_{3 k} x_{j}^{(m)}-b_{3 k}^{\prime}\right),\right. \\
& \left.x_{3 k-1}^{(m)}-E\left(\sum_{j=1}^{3 n^{2}} a_{3 k-1 j} x_{j}^{(m)}-b_{3 k-1}^{\prime}\right), \mu g_{k}\right) ; \\
& k=\frac{1, n^{2},}{m=0,1,2, \ldots},
\end{aligned}
$$

Сходимость итерационного процесса (28) к решению системы (24) будет иметь место независимо от выбора начального приближения $\left(x_{1}^{(0)}, x_{2}^{(0)}, \ldots, x_{3 n^{2}}^{(0)}\right.$ в $3 n^{2}$-мерном евклидовом пространстве $\mathbb{R}^{3 n^{2}}$ при условии, что матрица $A_{0}=$ $=\left(a_{i j}\right)_{i=1, j=1}^{3 n^{2}, 3 n^{2}}$ является симметрической и положительно определенной, а входящая в (28) положительная константа $E$ удовлетворяет неравенству

$$
E<\left(\max _{1 \leqslant i \leqslant 3 n^{2}} \sum_{j=1}^{3 n^{2}}\left|a_{i j}\right|\right)^{-1}
$$

Этот факт следует из принципа сжимающих отображений.

7. Численные результаты. Предложенным методом было получено численное решение пространственной квазистатической контактной задачи о взаимодействии упругого шара радиуса 0.3 м с упругим полупространством. Процесс нагружения тел осуществлялся в соответствии со следующим законом 
изменения жёстких смещений $\Delta_{1 i}, \Delta_{2 i}, \Delta_{3 i}$ шара, выраженных в метрах:

$$
\begin{aligned}
& \Delta_{1 i}=\left\{\begin{array}{r}
-0.000002 \cdot i, \quad \text { если } 1 \leqslant i \leqslant 50 ; \\
-0.0002+0.000002 \cdot i, \quad \text { если } 51 \leqslant i \leqslant 65 ;
\end{array}\right. \\
& \Delta_{2 i}=\Delta_{3 i}=0, \quad \text { если } 1 \leqslant i \leqslant 65 .
\end{aligned}
$$

График зависимости абсолютной величины нормального жесткого смещения шара $\left|\Delta_{1 i}\right|$ от номера шага нагружения $i$ представлен на рис. 2 . В соотношениях (29) величины $\Delta_{2 i}$ и $\Delta_{3 i}$ есть касательные составляющие жесткого смещения шара в направлении осей $O x$ и $O y$ соответственно.

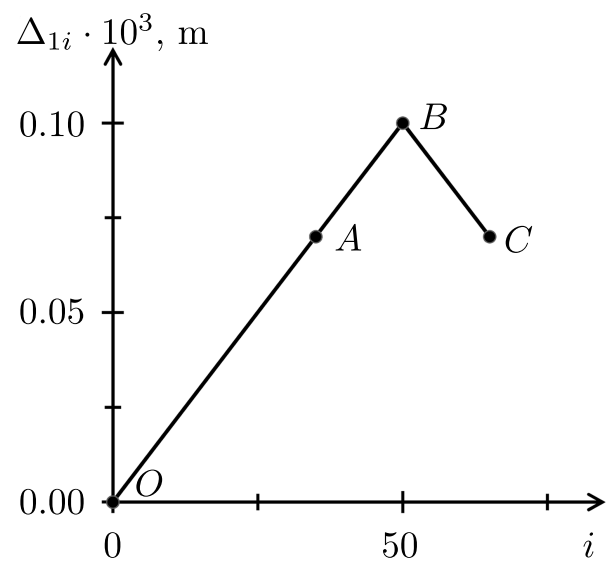

Рис. 2. Кривая нагружения [Figure 2. A loading curve]

В расчетах использовались следующие модули Юнга $E_{1}, E_{2}$ и коэффициенты Пуассона $\nu_{1}, \nu_{2}$ шара и полупространства: $E_{1}=3 \cdot 10^{5} \mathrm{MПа,} E_{2}=$ $=10^{5} \mathrm{MПа,} \nu_{1}=\nu_{2}=0.2$. Коэффициент трения $\mu=0.12375$. Значение безразмерного параметра $\beta$, определяемого согласно [10] формулой

$$
\beta=\frac{1}{2} \frac{E_{1}\left(1-2 \nu_{2}\right)\left(1+\nu_{2}\right)-E_{2}\left(1-2 \nu_{1}\right)\left(1+\nu_{1}\right)}{E_{1}\left(1-\nu_{2}^{2}\right)+E_{2}\left(1-\nu_{1}^{2}\right)},
$$

составляет для рассматриваемой контактной задачи 0.1875. Отношение $\mu / \beta=$ 0.66. При дискретизации зона контакта разбивалась на $41 \times 41=1681$ квадратных граничных элемента одинаковой площади $\left(n=41, \operatorname{mes}\left(\omega_{i}\right)=\right.$ $\left.=7.84 \cdot 10^{-8} \mathrm{M}^{2}\right)$. Для вычисления элементов $a_{k j}$ матрицы податливости использовались соотношения $(27)$, в которых функции $K_{j m}\left(s, s^{\prime}\right)$ задавались при помощи формул Буссинеска-Черрути, а значение $\varepsilon$ определялось соотношением

$$
\varepsilon=10^{-3} \cdot \int_{\omega_{k}} K_{11}\left(s_{j}^{n}, s\right) d s .
$$

С помощью итерационного процесса (28) в настоящей работе были получены два численных решения контактной задачи, которые соответствуют различным этапам процесса нагружения. Первое решение было получено на 
тридцать пятом шаге нагружения, что соответствует монотонному нормальному внедрению шара в полупространство на 0.00007 м (отрезок $O A$, рис. 2). Второе решение, полученное на последнем, шестьдесят пятом, шаге нагружения (ломаная $O B C$, рис. 2), соответствует первоначальному вдавливанию шара в полупространство на 0.0001 м (отрезок $O B$, рис. 2) и последующей разгрузке (отрезок $B C$, рис. 2). Отметим, что одноименные значения относительных жёстких смещений тел на тридцать пятом шаге совпали с их значениями на шестьдесят пятом шаге процесса нагружения тел.

Полученные из первого решения значения касательных напряжений $\tau_{z x}$, действующих в точках поверхности шара, соприкасающихся с полупространством по оси $O x$, сопоставлялись с результатами решения соответствующей осесимметричной контактной задачи, приведенными в работе [10] для случая $\mu / \beta=0.66$. В таблице показана зависимость безразмерной величины $\tau_{z x} /\left(\beta p_{\max }\right)$ от безразмерного параметра $x / a$.

Первый столбец таблицы соответствует решению [10], второй- численному решению, полученному на тридцать пятом шаге процесса нагружения (29), третий - численному решению, полученному на шестьдесят пятом шаге этого процесса. Здесь символ $p_{\max }$ обозначает максимальное значение контактного давления, а символ $a-$ радиус площадки контакта. Данные, приведенные в первом и втором столбцах таблицы, показывают хорошее соответствие решения [10] и численного решения, полученного при монотонном нагружении. Максимальная относительная погрешность в этом случае составляет приблизительно $6 \%$ по отношению к наибольшему значению величины $\tau_{z x} /\left(\beta p_{\max }\right)$, найденному согласно [10], что свидетельствует о корректности предложенного метода решения контактной задачи. Результаты, приведенные в третьем столбце таблицы, означают, что при разгрузке наблюдается уменьшение достигнутых значений касательных напряжений и смена знака этих значений вблизи границы пятна контакта.

Зависимость безразмерных касательных напряжений от безразмерного расстояния

[The dependence of the dimensionless shear stresses on the dimensionless distance]

\begin{tabular}{|c|c|c|c|}
\hline$x / a$ & Solution from [10] & $\begin{array}{c}\text { Solution by the Eqs. (28), (29) } \\
\text { at the step 35 }\end{array}$ & $\begin{array}{c}\text { Solution by the Eqs. }(28),(29) \\
\text { at the step } 65\end{array}$ \\
\hline 0.00 & 0.00 & 0.00 & 0.00 \\
0.06 & 0.10 & 0.07 & 0.07 \\
0.12 & 0.18 & 0.17 & 0.17 \\
0.18 & 0.25 & 0.22 & 0.23 \\
0.24 & 0.30 & 0.29 & 0.30 \\
0.30 & 0.35 & 0.35 & 0.35 \\
0.36 & 0.39 & 0.39 & 0.40 \\
0.42 & 0.42 & 0.42 & 0.43 \\
0.48 & 0.45 & 0.45 & 0.46 \\
0.54 & 0.47 & 0.47 & 0.48 \\
0.60 & 0.48 & 0.47 & 0.50 \\
0.66 & 0.48 & 0.46 & 0.48 \\
0.72 & 0.45 & 0.42 & 0.44 \\
0.78 & 0.40 & 0.37 & 0.40 \\
0.84 & 0.33 & 0.30 & 0.32 \\
0.90 & 0.23 & 0.22 & 0.12 \\
0.96 & 0.03 & 0.05 & -0.14 \\
\hline
\end{tabular}


На рис. 3 показаны конфигурации пятен контакта и зон сцепления, соответствующие полученным численным решениям. Здесь внешний замкнутый контур есть граница пятна контакта, заштрихованная область - зона сцепления, незаштрихованная область - зона проскальзывания. Рис. 3, а соответствует решению, полученному на тридцать пятом шаге, рис. $3, b$ - решению, полученному на шестьдесят пятом шаге процесса нагружения (29).

$$
y, \mathrm{~m}
$$

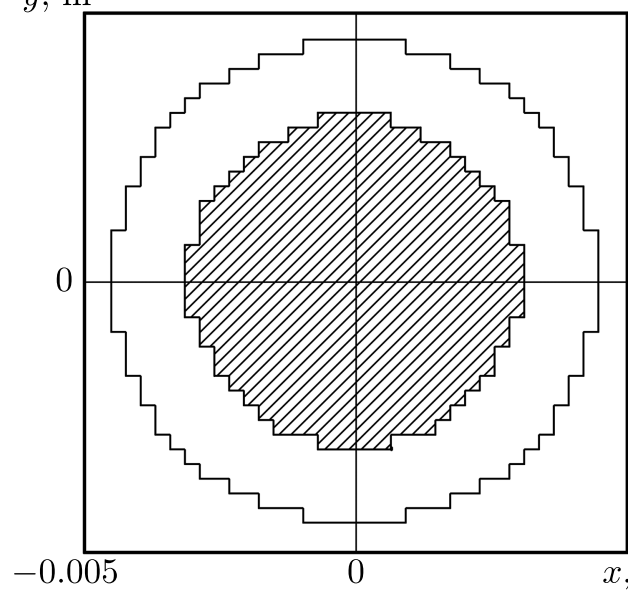

a

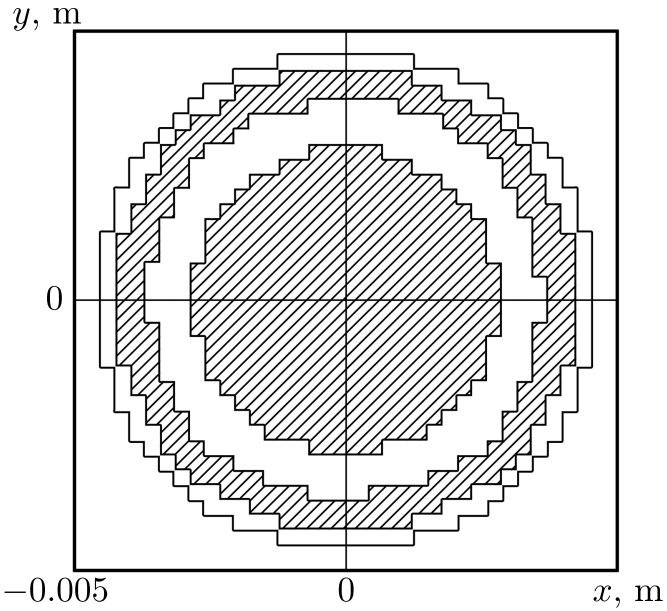

b

Рис. 3. Границы областей контакта и зон сцепления шара и полупространства при монотонном (a) и немонотонном (b) нагружении [Figure 3. The boundaries of the contact areas and the adhered regions of sphere and half-space under monotonic (a) and nonmonotonic (b) loading]

Анализ представленных на рис. 3 результатов показал, что качественные характеристики контакта тел невозможно установить по конечным значениям жёстких смещений этих тел, не учитывая, каким образом эти конечные значения были достигнуты в процессе нагружения. Полученные данные также показывают, что после прохождения этапа увеличения нагрузки до максимального значения (отрезок $A B$, рис. 2) и последующей разгрузки (отрезок $B C$, рис. 2) граница центрального участка зоны сцепления несколько сместилась к центру и одновременно вблизи границы пятна контакта образовалась вторая кольцевая зона сцепления. Отметим, что подобную конфигурацию зон сцепления на контактном пятне получил J. R. Turner [16] при исследовании случая разгрузки в задаче о взаимодействии цилиндрического штампа, имеющего плоскую подошву, с упругим полупространством.

Выводы. Разработан метод получения приближённого решения трёхмерной квазистатической контактной задачи о взаимодействии двух линейноупругих тел с учетом кулонова трения между ними при неизвестной заранее площадке контакта и неизвестной границе раздела зон проскальзывания и сцепления на этой площадке. Анализ полученных численных результатов для задачи о вдавливании упругого шара в упругое полупространство свидетельствует о корректности предложенного метода и его применимости к решению контактных задач рассматриваемого класса. 


\section{ORCID}

Юрий Михайлович Стреляев: http://orcid.org/0000-0002-4400-7824

\section{БИБЛИОГРАФИЧЕСКИЙ СПИСОК}

1. Кравчук А. С. Вариационный метод в контактных задачах. Состояние проблемы, направления развития // ПММ, 2009. Т. 73, № 3. С. 492-502.

2. Reina S. A., Dini D., Hills D. A., Lida Y. A quadratic programming formulation for the solution of layered elastic contact problems: Example applications and experimental validation // European Journal of Mechanics - A/Solids, 2011. vol.30, no.3. pp. 236-247. doi: 10.1016/j. euromechsol.2010.12.003.

3. Галанов Б. А. Метод граничных уравнений типа Гаммерштейна для контактных задач теории упругости в случае неизвестных областей контакта// ПMМ, 1985. Т. 49, № 5 . C. 827-835.

4. Галанов Б. А. О приближенном решении некоторых задач упругого контакта двух тел// Изв. АН СССР, МТT, 1981. № 5. С. 61-67.

5. Александров В. M., Kalker I. І., Пожарский Д. А. Пространственная контактная задача для двухслойного упругого основания с заранее неизвестной областью контакта // Изв. PAH, MTT, 1999. № 4. С. 51-55.

6. Чебаков М. И. Трехмерная контактная задача для слоя с учетом сил трения в области контакта// Изв. РАН, МТT, 2002. №6. С. 59-68.

7. Александров В. М., Пожарский Д. А. Трехмерные контактные задачи при учете трения и нелинейной шероховатости // ПММ, 2004. Т. 68, № 3. С. 516-527.

8. Александров А. И., Стреляев Ю. М. Метод нелинейных граничных интегральных уравнений для контактных задач теории упругости // Восточно-Европейский журнал передовых технологий, 2014. Т. 3, № 7(69). С. 36-40.

9. Александров А. И. Метод решения пространственной контактной задачи о взаимодействии двух упругих тел при наличии трения между ними // Mатематичні методи $i$ фізико-механічні поля, 2013. Т. 56, № 3. С. 29-42.

10. Johnson K. L. Contact Mechanics. Cambridge: Cambridge University Press, 1985, xii+452 pp. doi : $10.1017 /$ CB09781139171731.

11. Александров А. И. Неподвижные точки непрерывных операторов в гилъбертовом пространстве. Запорожье: Запорож. гос. ун-т, 2002. 77 с.

12. Тихонов А. Н., Арсенин В. Я. Методы решения некорректных задач. М.: Наука, 1986. 288 c.

13. Красносельский М. А., Вайникко Г. М., Забрейко П. П., Рутицкий Я. Б., Стеценко В. Я. Приближенное решение операторных уравнений. М.: Наука, 1969. 456 с.

14. Ахиезер Н. И., Глазман И. М. Теория линейных операторов в гилъбертовом пространстве. М.: Наука, 1966. 543 с.

15. Канторович Л. В., Акилов Г. П. Функииональный анализ. М.: Наука, 1984. 752 с.

16. Turner J. R. The frictional unloading problem on linear elastic half-space// IMA J. Appl. Math., 1979. vol. 24, no. 4. pp. 439-469. doi: 10.1093/imamat/24.4.439.

Поступила в редакцию 24/I/2016;

в окончательном варианте - $27 / \mathrm{IV} / 2016$;

принята в печать $-27 / \mathrm{V} / 2016$. 
Vestn. Samar. Gos. Techn. Un-ta. Ser. Fiz.-mat. nauki

[J. Samara State Tech. Univ., Ser. Phys. \& Math. Sci.], 2016, vol. 20, no. 2, pp. 306-327

ISSN: 2310-7081 (online), 1991-8615 (print)

doi: http://dx.doi.org/10.14498/vsgtu1471

MSC: 74M15, 74M10

\title{
A NONLINEAR BOUNDARY INTEGRAL EQUATIONS METHOD FOR THE SOLVING OF QUASISTATIC ELASTIC CONTACT PROBLEM WITH COULOMB FRICTION
}

\author{
Yu. M. Streliaiev \\ Zaporizhzhya National University, \\ 66, Zhukovskogo st., Zaporizhzhya, 69600, Ukraine.
}

\begin{abstract}
Three-dimensional quasistatic contact problem of two linearly elastic bodies' interaction with Coulomb friction taken into account is considered. The boundary conditions of the problem have been simplified by the modification of the Coulomb's law of friction. This modification is based on the introducing of a delay in normal contact tractions that bound tangent contact tractions in the Coulomb's law of friction expressions. At this statement the problem is reduced to a sequence of similar systems of nonlinear integral equations describing bodies' interaction at each step of loading. A method for an approximate solution of the integral equations system corresponded to each step of loading is applied. This method consists of system regularization, discretization of regularized system and iterative process application for solving the discretized system. A numerical solution of a contact problem of an elastic sphere with an elastic half-space interaction under increasing and subsequently decreasing normal compressive force has been obtained.
\end{abstract}

Keywords: elastic body, contact problem, Coulomb friction, quasistatic problem, integral equation, uniqueness of solution, regularizing equation, iterative process.

\section{ORCID}

Yurii M. Streliaiev: http://orcid.org/0000-0002-4400-7824

\section{REFERENCES}

1. Kravchuk A. S. The variational method in contact problems. The present state of the problem and trends in its development, J. Appl. Math. Mech., 2009, vol.73, no. 3, pp. 351-357. doi : $10.1016 / j$. jappmathmech.2009.07.004.

2. Reina S. A., Dini D., Hills D. A., Lida Y. A quadratic programming formulation for the solution of layered elastic contact problems: Example applications and experimental

(C) 2016 Samara State Technical University.

Please cite this article in press as:

Streliaiev Yu. M. A nonlinear boundary integral equations method for the solving of quasistatic elastic contact problem with Coulomb friction, Vestn. Samar. Gos. Tekhn. Univ., Ser. Fiz.-Mat. Nauki [J. Samara State Tech. Univ., Ser. Phys. \& Math. Sci.], 2016, vol. 20, no. 2, pp. 306-327. doi: 10.14498/vsgtu1471. (In Russian)

Author Details:

Yurii M. Streliaiev (strelkiny@gmail.com), Senior Lecturer, Dept. of Mathematical Analysis. 
validation, European Journal of Mechanics - A/Solids, 2011, vol.30, no.3, pp. 236-247. doi: 10.1016/j. euromechsol.2010.12.003.

3. Galanov B. A. The method of boundary equations of the Hammerstein-type for contact problems of the theory of elasticity when the regions of contact are not known, J. Appl. Math. Mech., 1985, vol.49, no. 5, pp. 634-640. doi: 10.1016/0021-8928(85)90084-X.

4. Galanov B. A. Approximate solution to some problems of elastic contact of two bodies, Mech. Solids, 1981, vol. 16, no. 5, pp. 61-67.

5. Alexandrov V. M., Kalker J. J., Pozharskii D. A. Three-dimensional contact problem for a two-layered elastic base with an unknown contact area, Mech. Solids, 1999, vol. 34, no. 4, pp. $41-45$.

6. Chebakov M. I. 3D contact problem with friction for a layer, Mech. Solids, 2002, vol.37, no. 6 , pp. $48-56$.

7. Aleksandrov V. M., Pozharskii D. A. Three-dimensional contact problems taking friction and non-linear roughness into account, J. Appl. Math. Mech., 2004, vol.68, no. 3, pp. 463472. doi: 10.1016/S0021-8928(04)00061-9.

8. Aleksandrov A. I., Streliaiev Yu. M. Nonlinear boundary integral equations method for contact problems of the elasticity theory, Eastern-European Journal of Enterprise Technologies, 2014, vol. 3, no. 7(69), pp. 36-40 (In Russian).

9. Aleksandrov A. I. Method of solution of three-dimensional contact problem on interaction of two elastic bodies in the presence of friction between them, Matematichni metodi i fizikomekhanichni polia, 2013, vol.56, no. 3, pp. 29-42 (In Russian).

10. Johnson K. L. Contact Mechanics. Cambridge, Cambridge University Press, 1985, xii+452 pp. doi: 10.1017/CB09781139171731.

11. Aleksandrov A. I. Nepodvizhnye tochki nepreryvnykh operatorov v gil'bertovom prostranstve [Fixed points of continuous operators in the Hilbert space]. Zaporozhye, Zaporozh. State Univ., 2002, 77 pp. (In Russian)

12. Tikhonov A. N., Arsenin V. Yu. Solutions of ill-posed problems, Scripta Series in Mathematics. New York, John Wiley \& Sons, 1977, xiii+258 pp.

13. Krasnosel'skij M. A., Vainikko G. M., Zabreiko P. P. Rutitskij Ya. B., Stetsenko V. Ya. Approximate solution of operator equations, Wolters-Noordhoff Series of Monographs and Textbooks on Pure and Applied Mathematics. Groningen, Wolters-Noordhoff Publ., 1972, xii+484 pp.

14. Akhiezer N. I., Glazman I. M. Theory of linear operators in Hilbert space, vol. I, II, Monographs and Studies in Mathematics, vol. 9, 10. Edinburgh, Scottish Academic Press, 1981.

15. Kantorovich L. V., Akilov G. P. Functional analysis. Oxford, Pergamon Press, 1982, xiv+589 pp.

16. Turner J. R. The frictional unloading problem on linear elastic half-space, IMA J. Appl. Math., 1979, vol. 24, no. 4, pp. 439-469. doi: 10.1093/imamat/24.4.439.

Received 24/I/2016;

received in revised form $27 / \mathrm{IV} / 2016$;

accepted $27 / \mathrm{V} / 2016$. 